\title{
Lateral Domain of the Epithelium
}

National Cancer Institute

\section{Source}

National Cancer Institute. Lateral Domain of the Epithelium. NCI Thesaurus. Code C32937.

The non-free sides of an epithelial cell that connect to other epithelial cells when forming the epithelium. 